\title{
A FATOU-TYPE THEOREM FOR HARMONIC FUNCTIONS ON SYMMETRIC SPACES ${ }^{1}$
}

\author{
BY S. HELGASON AND A. KORÁNYI
}

Communicated by G. D. Mostow, August 31, 1967

1. Introduction. The result to be proved in this article is that if $u$ is a bounded harmonic function on a symmetric space $X$ and $x_{0}$ any point in $X$ then $u$ has a limit along almost every geodesic in $X$ starting at $x_{0}$ (Theorem 2.3). In the case when $X$ is the unit disk with the nonEuclidean metric this result reduces to the classical Fatou theorem (for radial limits). When specialized to this case our proof is quite different from the usual one; in fact it corresponds to transforming the Poisson integral of the unit disk to that of the upper half-plane and using only a homogeneity property of the Poisson kernel. The kernel itself never enters into the proof.

2. Harmonic functions on symmetric spaces. Let $G$ be a semisimple connected Lie group with finite center, $K$ a maximal compact subgroup of $G$ and $\mathfrak{g}$ and $\mathfrak{t}$ their respective Lie algebras. Let $B$ denote the Killing form of $\mathfrak{g}$ and $\mathfrak{p}$ the corresponding orthogonal complement of $\mathfrak{l}$ in $\mathfrak{g}$. Let Ad denote the adjoint representation of $G$. As usual we view $\mathfrak{p}$ as the tangent space to the symmetric space $X=G / K$ at the origin $o=\{K\}$ and accordingly give $X$ the $G$-invariant Riemannian structure induced by the restriction of $B$ to $\mathfrak{p} \times \mathfrak{p}$. Let $\Delta$ denote the corresponding Laplace-Beltrami operator.

Fix a maximal abelian subspace $\mathfrak{a} \subset \mathfrak{p}$ and let $M$ denote the centralizer of $\mathfrak{a}$ in $K$. If $\lambda$ is a linear function on $\mathfrak{a}$ and $\lambda \neq 0$ let $\mathfrak{g}_{\lambda}$ $=\{X \in \mathfrak{g} \mid[H, X]=\lambda(H) X$ for all $H \in \mathfrak{a}\} ; \lambda$ is called a restricted root if $\mathfrak{g}_{\lambda} \neq 0$. Let $\mathfrak{a}^{\prime}$ denote the open subset of $\mathfrak{a}$ where all restricted roots are $\neq 0$. Fix a Weyl chamber $\mathfrak{a}^{+}$in $\mathfrak{a}$, i.e. a connected component of $a^{\prime}$. A restricted root $\alpha$ is called positive (denoted $\alpha>0$ ) if its values on $\mathfrak{a}^{+}$are positive. Let the linear function $\rho$ on $\mathfrak{a}$ be determined by $2 \rho=\sum_{\alpha>0}\left(\operatorname{dim} \mathfrak{g}_{\alpha}\right) \alpha$ and denote the subalgebras $\sum_{\alpha>0} \mathfrak{g}_{\alpha}$ and $\sum_{\alpha>0} \mathfrak{g}_{-\alpha}$ of $\mathfrak{g}$ by $\mathfrak{n}$ and $\overline{\mathfrak{n}}$ respectively. Let $N$ and $\bar{N}$ denote the corresponding analytic subgroups of $G$.

By a Weyl chamber in $\mathfrak{p}$ we understand a Weyl chamber in some maximal abelian subspace of $\mathfrak{p}$. The boundary of $X$ is defined as the set $B$ of all Weyl chambers in the tangent space $p$ to $X$ at $o$; since this boundary is via the map $k M \rightarrow \operatorname{Ad}(k) a^{+}$identified with $K / M$, which by the Iwasawa decomposition $G=K A N$ equals $G / M A N$, this defi-

${ }^{1}$ This work was supported by the National Science Foundation, GP 7477 and GP 6155. 
nition of boundary is equivalent to Furstenberg's [2] (see also [6] and [4]). In particular the group $G$ acts transitively on $B$ as well as on $X$. The two actions will be denoted $(g, b) \rightarrow g(b)$ and $(g, x) \rightarrow g \cdot x$ $(g \in G, b \in B, x \in X)$. Let $d b$ denote the unique $K$-invariant measure on $B$ normalized by $\int_{B} d b=1$. Then according to Furstenberg [2], the mapping $f \rightarrow u$ where

$$
u(g \cdot o)=\int_{B} f(g(b)) d b \quad(g \in G),
$$

is a bijection of the set $L^{\infty}(B)$ of bounded measurable functions on $B$ onto the set of bounded solutions of Laplace's equation $\Delta u=0$ on $X$. The function $u$ in (1) is called the Poisson integral of $f$.

If $g \in G$ let $k(g) \in K, H(g) \in \mathfrak{a}$ be determined by $g=k(g) \exp H(g) n$ $(n \in N)$. Observe that if $g^{h}$ denotes $h g h^{-1}$ for $h \in G$ then $k\left(\bar{n}^{m}\right)=k(\bar{n})^{m}$, $H\left(\bar{n}^{m}\right)=H(\bar{n})$ for $\bar{n} \in \bar{N}, m \in M$. According to Harish-Chandra [3, Lemma 44], the mapping $\bar{n} \rightarrow k(\bar{n}) M$ is a bijection of $\bar{N}$ onto a subset of $K / M$ whose complement is of lower dimension and if $f$ is a continuous function on $B$, then

$$
\int_{B} f(b) d b=\int_{\bar{N}} f(k(\bar{n}) M) \exp (-2 \rho(H(\bar{n}))) d \tilde{n}
$$

for a suitably normalized Haar measure $d \bar{n}$ on $\bar{N}$. If $a \in A$ we have $a k(\bar{n}) M A N=k\left(\bar{n}^{a}\right) M A N$ whence

$$
a(k(\bar{n}) M)=k\left(\bar{n}^{a}\right) M
$$

so the action of $a$ on the boundary corresponds to the conjugation $\bar{n} \rightarrow \bar{n}^{a}$ on $\bar{N}$.

Let $E_{1}, \cdots, E_{r}$ be a basis of $\overline{\mathfrak{n}}$ such that each $E_{i}$ lies in some $\mathfrak{g}_{-\alpha}$, say $\mathfrak{g}_{-\alpha_{\mathfrak{i}}}$. Since the map exp: $\overline{\mathfrak{n}} \rightarrow \bar{N}$ is a bijection we can, for each $H \in \mathfrak{a}^{+}$, consider the function $\bar{n} \rightarrow|\bar{n}|_{H}$ defined as follows: If $\bar{n}=\exp \left(\sum_{1}^{r} a_{i} E_{i}\right)\left(a_{i} \in R\right)$ we put

$$
|\bar{n}|_{H}=\operatorname{Max}_{1 \leqq ı \leq r}\left(\left|a_{i}\right|^{1 / a_{i}(H)}\right)
$$

Since

we have

$$
\bar{n}^{\exp t H}=\exp \left(\sum_{1}^{r} a_{i} \exp \left(-\alpha_{i}(H) t\right) E_{i}\right)
$$

$$
\left|\bar{n}^{\exp t \theta}\right|_{H}=e^{-t}|\bar{n}|_{H} \quad \text { for } \bar{n} \in \bar{N}, \quad t \in R, \quad H \in \mathfrak{a}^{+} .
$$

For $r>0$ let $B_{H, r}$ denote the set $\left\{\left.\bar{n} \in \bar{N}|| \bar{n}\right|_{H}<r\right\}$ and let $V_{H, r}$ denote the volume of $B_{H, r}$ (with respect to the Haar measure on $\bar{N}$ ). 
Lemma 2.1. Let $f \in L^{\infty}(B)$ and $u$ the Poisson integral (1) of $f$. Put $F(\bar{n})=f(k(\bar{n}) M)$ for $\bar{n} \in \bar{N}$. Fix $\bar{n}_{0} \in \bar{N}$ and $H \in \mathfrak{a}^{+}$and assume

$$
\frac{1}{V_{H, r}} \int_{B_{H, r}}\left|F\left(\bar{n}_{0} \bar{n}\right)-F\left(\bar{n}_{0}\right)\right| d \bar{n} \rightarrow 0
$$

for $r \rightarrow 0$. Then

$$
\lim _{t \rightarrow+\infty} u\left(k\left(\bar{n}_{0}\right) \exp t H(\cdot 0)\right)=f\left(k\left(\bar{n}_{0}\right) M\right) .
$$

Proof. By the Iwasawa decomposition we can write $\bar{n}_{0}=k\left(\bar{n}_{0}\right)$ $\cdot\left(a_{1} n_{1}\right)^{-1}\left(a_{1} \in A, n_{1} \in N\right)$ so

$$
u\left(k\left(\bar{n}_{0}\right) \exp t H \cdot o\right)=u\left(\bar{n}_{0} a_{1} n_{1} \exp t H \cdot o\right)=u\left(\bar{n}_{0} \exp t H a_{1} n_{1}^{\exp (-t H) \cdot o)}\right.
$$

But $G=A \bar{N} K$ so $n_{1}^{\exp (-t H)}=a(t) \bar{n}(t) k(t)$, each factor tending to $e$ as $t \rightarrow+\infty$. If $H_{t} \in \mathfrak{a}$ is determined by

$$
\exp t H_{t}=\exp t H a_{1} a(t)
$$

we have

$$
u\left(k\left(\bar{n}_{0}\right) \exp t H \cdot o\right)=u\left(\bar{n}_{0} \bar{n}(t)^{\exp t H_{t}} \exp t H_{t} \cdot 0\right) .
$$

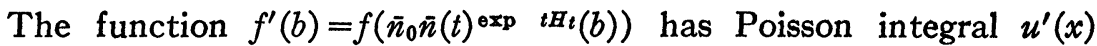
$=u\left(\bar{n}_{0} \bar{n}(t)^{\exp t H_{t}} \cdot x\right)$; using (1) on $u^{\prime}$ and $f^{\prime}$ with $g=\exp t H_{t}$ we get from (2) and (3)

$u\left(k\left(\bar{n}_{0}\right) \exp t H \cdot o\right)-f\left(k\left(\bar{n}_{0}\right) M\right)$

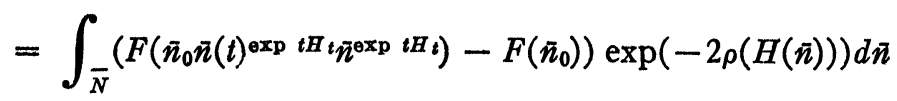

so

$$
\begin{aligned}
\mid u\left(k\left(\bar{n}_{0}\right)\right. & \exp t H \cdot o)-f\left(k\left(\bar{n}_{0}\right) M\right) \mid \\
& \leqq \int_{\bar{N}}\left|F\left(\bar{n}_{0} \bar{n}^{\exp t H_{t}}\right)-F\left(\bar{n}_{0}\right)\right| \exp \left(-2 \rho\left(H\left(\bar{n}(t)^{-1} \bar{n}\right)\right)\right) d \bar{n} .
\end{aligned}
$$

Now if $c>0$ let $\bar{N}_{c}$ denote the "square"

$$
\bar{N}_{c}=\left\{\exp \left(\sum_{1}^{r} a_{i} E_{i}\right)|| a_{i} \mid \leqq c, 1 \leqq i \leqq r\right\} \text {. }
$$

The integral on the right in (7) equals the sum 


$$
\int_{\bar{N}_{c}}\left|F\left(\bar{n}_{0} \bar{n}^{\exp t H_{t}}\right)-F\left(\bar{n}_{0}\right)\right| \exp \left(-2 \rho\left(H\left(\bar{n}(t)^{-1} \bar{n}\right)\right)\right) d \bar{n}
$$

$$
+\int_{\bar{N}-\bar{N}_{a}}\left|F\left(\bar{n}_{0} \bar{n}^{\exp t H_{t}}\right)-F\left(\bar{n}_{0}\right)\right| \exp \left(-2 \rho\left(H\left(\bar{n}(t)^{-1} \bar{n}\right)\right)\right) d \bar{n} .
$$

Since $\rho(H(\bar{n})) \geqq 0$ for all $\bar{n} \in \bar{N}([3$, p. 287]) and since the mapping $\bar{n} \rightarrow \bar{n}^{\exp H}$ has Jacobian $\exp (-2 \rho(H))$ (cf. (4)) we see that

$$
\int_{\bar{N}_{c}}\left|F\left(\bar{n}_{0} \bar{n}^{\exp t H}\right)-F\left(\bar{n}_{0}\right)\right| \exp \left(-2 \rho\left(H\left(\bar{n}(t)^{-1} \bar{n}\right)\right)\right) d \bar{n}
$$

$$
\leqq \exp \left(2 \rho\left(t H_{t}\right)\right) \int_{\bar{N}_{c}^{\exp t H_{t}}}\left|F\left(\bar{n}_{0} \bar{n}\right)-F\left(\bar{n}_{0}\right)\right| d \bar{n} .
$$

Now $\bar{n} \in \bar{N}_{c} \exp t B_{t}$ if and only if

$$
\bar{n}=\exp \left(\sum a_{i} e^{-\alpha_{i}\left(t H_{t}\right)} E_{i}\right) \quad \text { where }\left|a_{i}\right| \leqq c
$$

and $t H_{t}-t H$ is bounded (for fixed $\bar{n}_{0}$ and $H$ ). It follows that

$$
\bar{N}_{c}^{\text {exp } t H_{t}} \subset B_{H, d e^{-t}} \quad \text { for all } t \geqq 0 \text {, }
$$

$d=d\left(H, \bar{n}_{0}, c\right)$ being a constant. But since the map exp: $\overline{\mathfrak{n}} \rightarrow \bar{N}$ is measure-preserving it is clear that

$$
V_{H, d e^{-t}}=\exp (-2 \rho(H) t) d_{1} \quad t \geqq 0
$$

where $d_{1}=d_{1}\left(H, \bar{n}_{0}, c\right)$ is another constant. Also

$$
\exp \left(2 \rho\left(t H_{t}\right)\right) \leqq \exp (2 \rho(t H)) d_{2}
$$

where $d_{2}\left(H, \bar{n}_{0}\right)$ is a constant. Thus the right hand side of (9) can be majorized for all $t \geqq 0$ :

$$
\begin{aligned}
\exp 2 \rho\left(t H_{t}\right) \int_{\bar{N}_{c} \exp t H_{t}} \mid & F\left(\bar{n}_{0} \bar{n}\right)-F\left(\bar{n}_{0}\right) \mid d \bar{n} \\
& \leqq d_{3} \frac{1}{V_{H, d e^{-t}}} \int_{B_{H, d e}-t}\left|F\left(\bar{n}_{0} \bar{n}\right)-F\left(\bar{n}_{0}\right)\right| d \bar{n}
\end{aligned}
$$

where $d$ and $d_{3}$ are constants depending on $H, \bar{n}_{0}$ and $c$.

On the other hand, if \|\|$_{\infty}$ denotes the uniform norm on $\bar{N}$ the second term in (8) is majorized by

$$
\begin{aligned}
2\|F\|_{\infty} \int_{\bar{N}_{-} \bar{N}_{c}} \exp \left(-2 \rho\left(H\left(\bar{n}(t)^{-1} \bar{n}\right)\right)\right) d \bar{n} \\
=2\|F\|_{\infty}\left(1-\int_{\bar{n}(t) \bar{N}_{c}} \exp (-2 \rho(H(\bar{n}))) d \bar{n}\right) .
\end{aligned}
$$


Now given $\epsilon>0$ we first choose $c$ so large that

$$
2\|F\|_{\infty}\left(1-\int_{\bar{N}_{c / 2}} \exp (-2 \rho(H(\bar{n}))) d \bar{n}\right)<\epsilon / 2 ;
$$

since $\bar{n}(t) \rightarrow e$ for $t \rightarrow+\infty$ we can choose $t_{1}$ such that $\bar{n}(t) \bar{N}_{c} \supset \bar{N}_{c / 2}$ for $t \geqq t_{1}$. Then the expression in (11) is $<\epsilon / 2$ for $t \geqq t_{1}$; by our assumption (6) we can choose $t_{2}$ such that the right hand side of (10) is $<\epsilon / 2$ for $t>t_{2}$. In view of (7) and (8) this proves the lemma.

The next lemma shows that, for a fixed $H$, the assumption of Lemma 2.1 actually holds for almost all $\bar{n}_{0} \in \bar{N}$.

Lemma 2.2. Let $F \in L^{\infty}(\bar{N})$ and $f i x H \in \mathfrak{a}^{+}$. Then

$$
\lim _{r \rightarrow 0} \frac{1}{V_{H, r}} \int_{B_{H, r}}\left|F\left(\bar{n}_{0} \bar{n}\right)-F\left(\bar{n}_{0}\right)\right| d \bar{n}=0
$$

for almost all $\bar{n}_{0} \in \bar{N}$.

The proof of this result is essentially in the literature: In [1] Edwards and Hewitt give all the necessary arguments for the case of a discrete sequence tending to 0 and everything they do remains trivially valid in the case $r \rightarrow 0$. The result in the exact form required here was also proved by E. M. Stein independently of [1] (cf. his expository article [6]).

THEOREM 2.3. Let $u$ be a bounded solution of Laplace's equation $\Delta u=0$ on the symmetric space $X$. Then for almost all geodesics $\gamma(t)$ starting at $o$

$$
\lim _{t \rightarrow \infty} u(\gamma(t)) \text { exists. }
$$

Proof. Let $S^{+}=\left\{H \in \mathfrak{a}^{+} \mid B(H, H)=1\right\}$. Then the mapping $(k M, H) \rightarrow \operatorname{Ad}(k) H$ is a bijection of $(K / M) \times S^{+}$onto a subset of the unit sphere $S$ in $\mathfrak{p}$ whose complement has lower dimension. Since $\operatorname{dim}(K / M-k(\bar{N}) / M)<\operatorname{dim} K / M$ the mapping $(\bar{n}, H) \rightarrow \operatorname{Ad}(k(\bar{n})) H$ is a bijection of $\bar{N} \times S^{+}$onto a subset of $S$ whose complement in $S$ has lower dimension. If $\bar{N}_{H}$ denotes the set of $\bar{n}_{0}$ for which (12) holds (with $F(\bar{n})=f(k(\bar{n}) M)$ ) and if $S_{0}=\bigcup_{H \in S^{+}} \operatorname{Ad}\left(k\left(\bar{N}_{H}\right)\right) H$ it follows from the Fubini theorem that $S-S_{0}$ is a null set. This concludes the proof.

REMARKS. (i) If $f$ is continuous the limit relation

$$
\lim _{t \rightarrow+\infty} u(k \exp t H \cdot o)=f(k M) \quad\left(H \in \mathfrak{a}^{+}, k M \in K / M\right)
$$


follows immediately from (1), (2) and (3), by use of the dominated convergence theorem. (See also [4, Theorem 18.3.2.]) In particular, $u$ has the same limit along all geodesics from $o$ which lie in the same Weyl chamber in $\mathfrak{p}$.

(ii) In the case when $X$ has rank one $(\operatorname{dim} \mathfrak{a}=1)$ A. W. Knapp [5] has proved (13), even under the weaker assumption that $f \in L^{1}(B)$.

\section{REFERENCES}

1. R. E. Edwards and E. Hewitt, Pointwise limits for sequences of convolution operators, Acta Math. 113 (1965), 181-218.

2. H. Furstenberg, A Poisson formula for semisimple Lie groups, Ann. of Math. 77 (1963), 335-386.

3. Harish-Chandra, Spherical functions on a semisimple Lie group. I, Amer. J. Math. 80 (1958), 241-310.

4. F. I. Karpelevic, Geometry of geodesics and eigenfunctions of the Laplace-Beltrami operator on symmetric spaces, Trudy Moscov. Mat. Obšc. 14 (1965), 48-185.

5. A. W. Knapp, unpublished manuscript.

6. C. Moore, Compactifications of symmetric spaces, Amer. J. Math. 86 (1964), 201-218.

7. E. M. Stein, Maximal functions and Fatou's theorem, C.I.M.E. summer course on bounded homogeneous domains, Cremonese, 1967.

Massachusetrs Institute of Technology and YeshIVA UNIVERSITY 